\title{
Realizing high comprehensive energy storage and ultrahigh hardness in lead-free ceramics
}

Jie Xinga, Yanli Huang, ${ }^{\mathrm{a}}$, Qian $\mathrm{Xu}^{\mathrm{b}}$, Bo Wu $\mathrm{Wu}^{\mathrm{c}}$, Qiming Zhang ${ }^{\mathrm{d}}$, Zhi Tan ${ }^{\mathrm{a}, *}$, Qiang Chen ${ }^{\mathrm{a}}$, Jiagang $\mathrm{Wu}^{\mathrm{a}}$, and Jianguo $\mathrm{Zhu}^{\mathrm{a}, *}$

a College of Materials Science and Engineering, Sichuan University, 610064 Chengdu, China

b Key Laboratory of Deep Underground Science and Engineering (Ministry of Education), College of Architecture and Environment, Sichuan University, Chengdu, 610065, China

c Sichuan Province Key Laboratory of Information Materials, Southwest Minzu University, 610041, Chengdu, China

d Department of Physics, University of Texas at Arlington, TX 76019, USA

* Corresponding authors: tanzhi0838@163.com

* Corresponding authors: nic0400@scu.edu.cn 
Table S1 Mechanical properties of KNN- $x \mathrm{LB}$ ceramics derived from Vickers indentation test

\begin{tabular}{llll}
\hline & $\mathbf{P}(\mathbf{N})$ & $\mathbf{d}(\boldsymbol{\mu m})$ & $\boldsymbol{H}(\mathbf{G P a})$ \\
\hline $\mathbf{0 . 0 2 0}$ & 4.903 & 37.60 & 6.43 \\
$\mathbf{0 . 0 2 5}$ & 4.903 & 37.03 & 6.63 \\
$\mathbf{0 . 0 3 0}$ & 4.903 & 40.76 & 5.47 \\
$\mathbf{0 . 0 3 5}$ & 4.903 & 41.90 & 5.18 \\
\hline
\end{tabular}
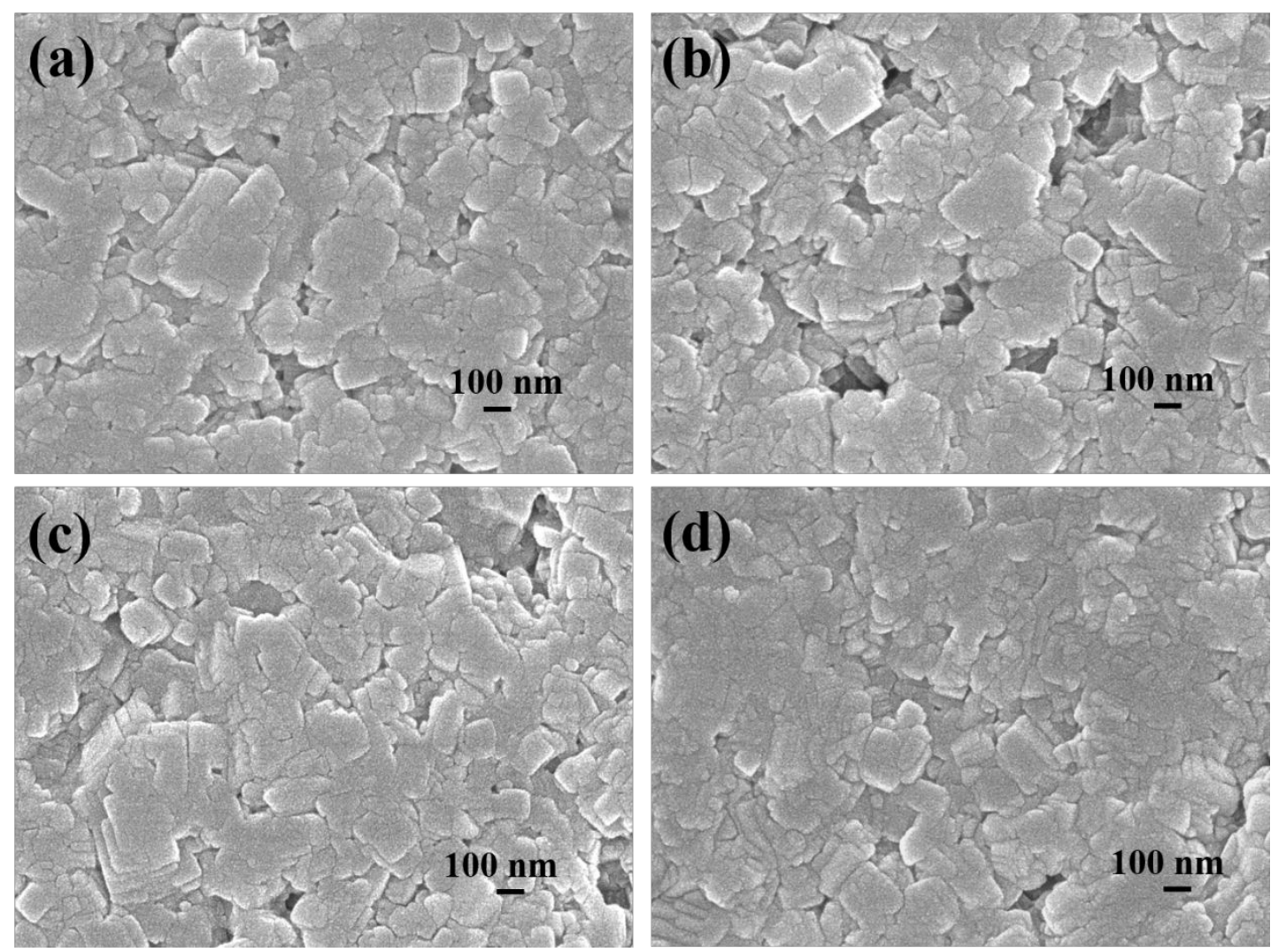

Fig.S1 SEM images of KNN- $x$ BL ceramics (a) $x=0.020$; (b) $x=0.025$; (c) $x=0.030$; (d) $x=0.035$ 
Table S2 Crystal structure parameters of KNN- $x \mathrm{BL}$ ceramics derived from the Rietveld structure refinement program

\begin{tabular}{|c|c|c|c|c|c|}
\hline composition & \multicolumn{2}{|c|}{ lattice parameters } & $\Delta$ & $\operatorname{sig}$ & $R_{\mathrm{w}}(\%)$ \\
\hline \multirow{3}{*}{$x=0.02$} & $a=5.6164 \AA$ & $\mathrm{a}_{\mathrm{c}}=3.9923 \AA$ & \multirow{3}{*}{0.0265} & \multirow{3}{*}{1.59} & \multirow{3}{*}{3.80} \\
\hline & $b=3.9658 \AA$ & $b_{c}=3.9658 \AA$ & & & \\
\hline & $c=5.6755 \AA$ & $c_{c}=3.9923 \AA$ & & & \\
\hline \multirow{3}{*}{$x=0.025$} & $a=5.6197 \AA$ & $a_{c}=3.9900 \AA$ & \multirow{3}{*}{0.0226} & \multirow{3}{*}{1.52} & \multirow{3}{*}{3.90} \\
\hline & $b=3.9673 \AA$ & $b_{c}=3.9673 \AA$ & & & \\
\hline & $\mathrm{c}=5.6655 \AA$ & $\mathrm{c}_{\mathrm{c}}=3.9900 \AA$ & & & \\
\hline \multirow{3}{*}{$x=0.03$} & $\mathrm{a}=5.6071 \AA$ & $\mathrm{a}_{\mathrm{c}}=3.9866 \AA$ & \multirow{3}{*}{0.0253} & \multirow{3}{*}{1.87} & \multirow{3}{*}{4.58} \\
\hline & $b=3.9613 \AA$ & $b_{c}=3.9613 \AA$ & & & \\
\hline & $\mathrm{c}=5.6686 \AA$ & $\mathrm{c}_{\mathrm{c}}=3.9866 \AA$ & & & \\
\hline \multirow{3}{*}{$x=0.035$} & $a=5.6178 \AA$ & $a_{c}=3.9876 \AA$ & \multirow{3}{*}{0.0322} & \multirow{3}{*}{1.51} & \multirow{3}{*}{3.68} \\
\hline & $b=3.9677 \AA$ & $b_{c}=3.9677 \AA$ & & & \\
\hline & $\mathrm{c}=5.6952 \AA$ & $c_{c}=3.9876 \AA$ & & & \\
\hline
\end{tabular}
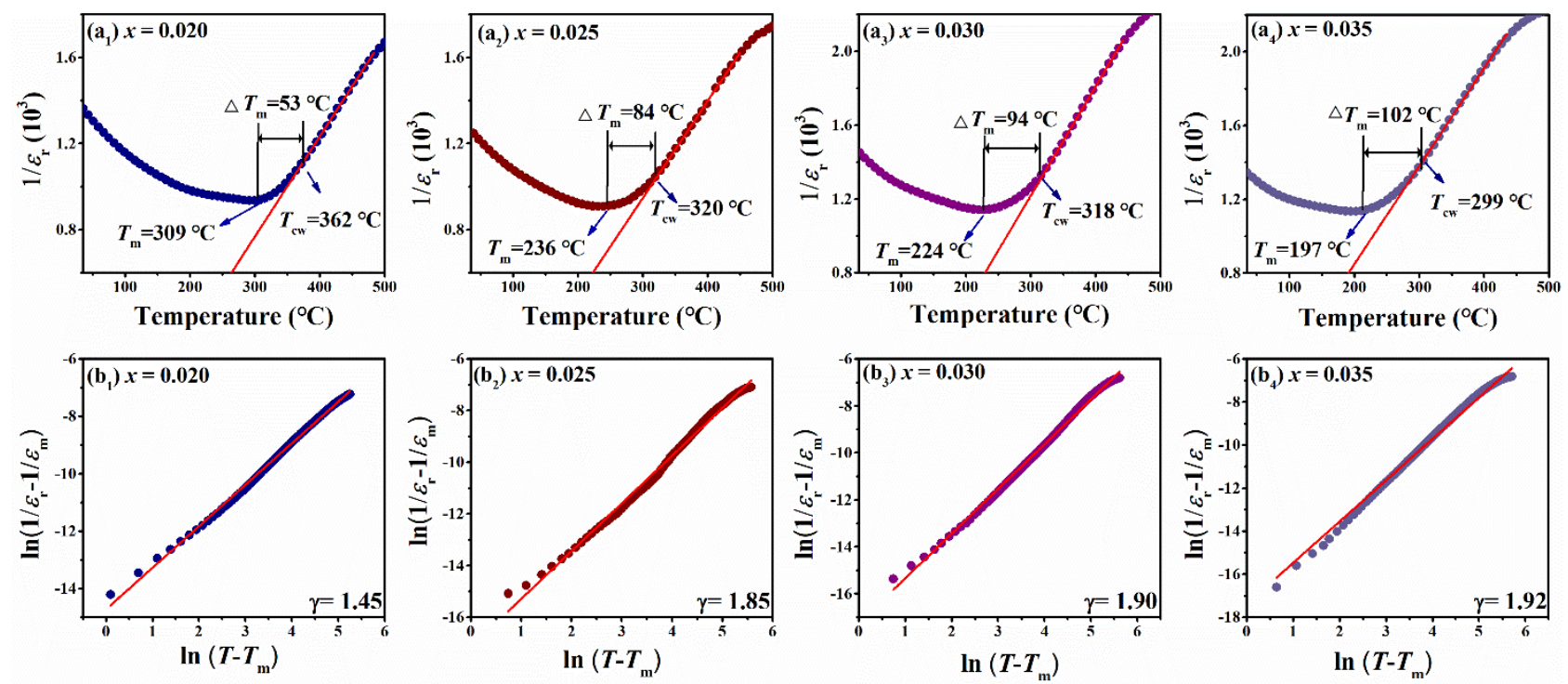

Fig. S2 $\left(a_{1}\right)-\left(a_{4}\right)$ Inverse dielectric permittivity as a function of temperature; $\left(b_{1}\right)-\left(b_{4}\right)$ the plots of $\log \left(1 / \varepsilon_{\mathrm{r}}-\right.$ $\left.1 / \varepsilon_{\mathrm{m}}\right)$ as a function of $\log \left(T-T_{\mathrm{m}}\right)$ for KNN-xLB ceramics [symbol: experimental data; solid line: linear fitting] 


\section{$x=0.025$}

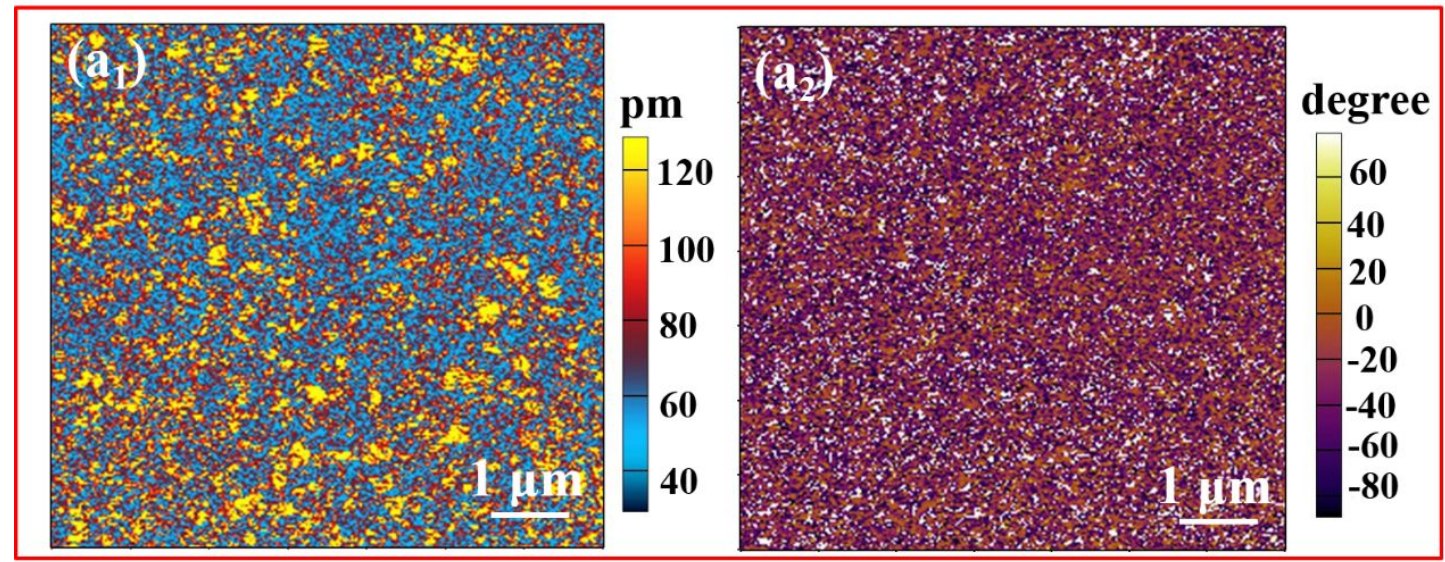

$x=\mathbf{0 . 0 3 0}$

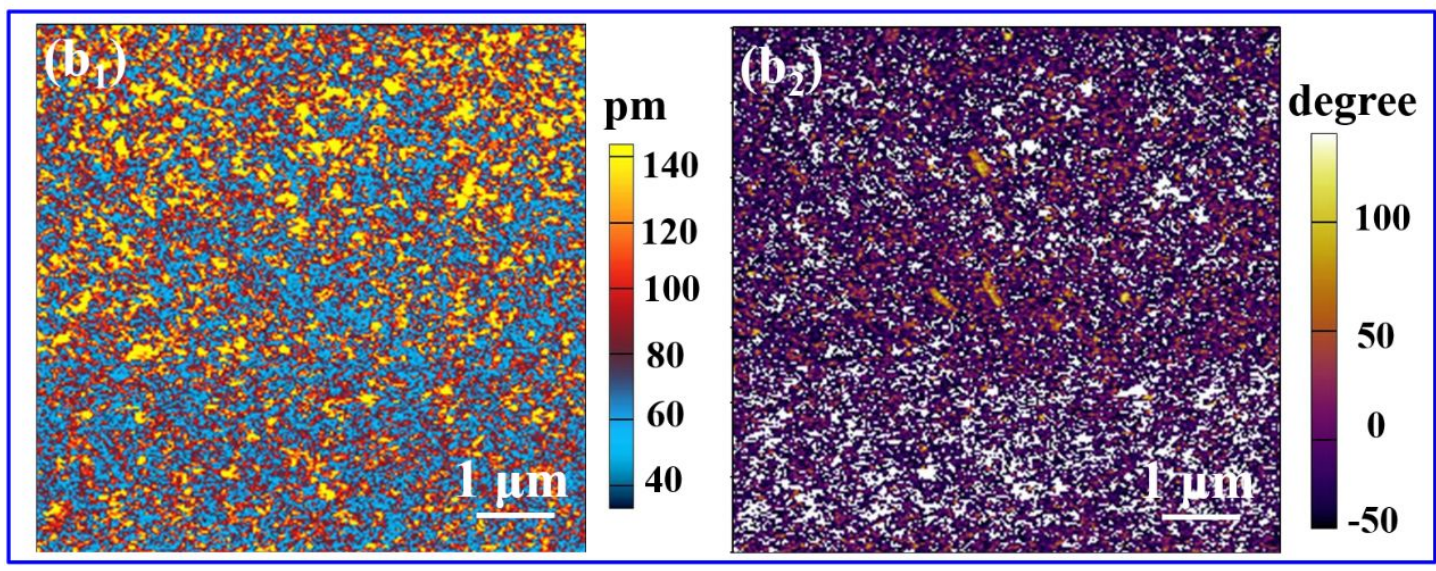

Fig. S3 Vertical piezoelectric force microscopy (VPFM) images for ceramic with $x=0.025$ and $x=0.030$ : $\left(a_{1}\right)$,

$\left(b_{1}\right)$ amplitude patterns and $\left(a_{2}\right),\left(b_{2}\right)$ phase patterns 


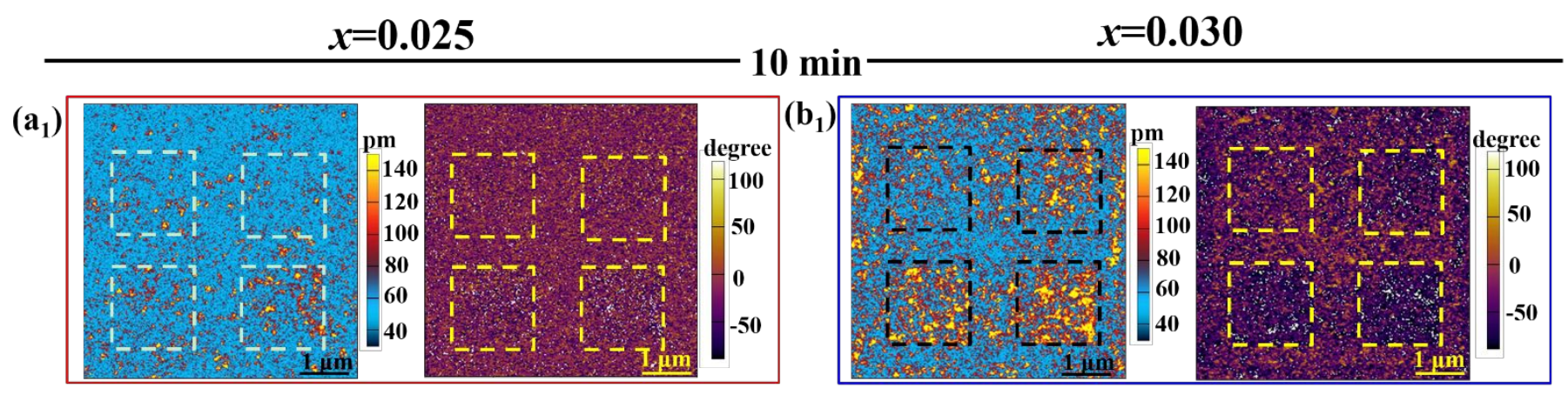

$30 \mathrm{~min}$

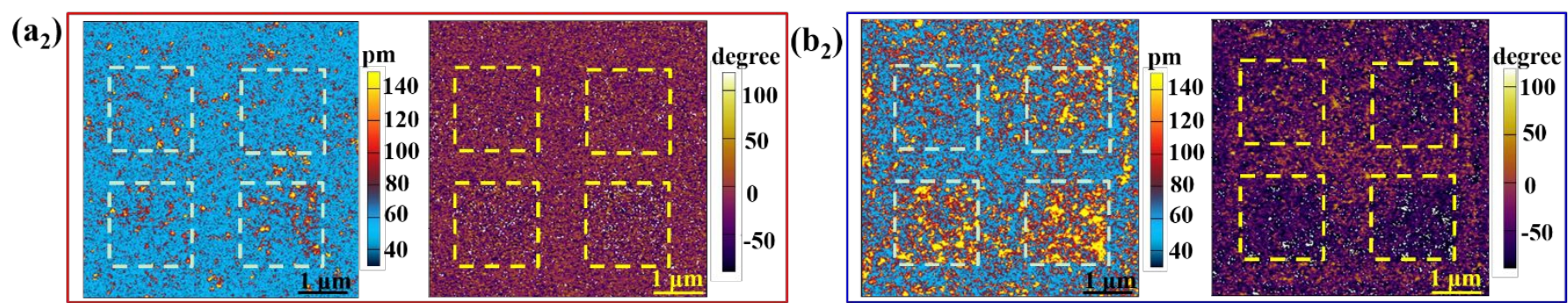

Fig. S4 Vertical piezoresponse force microscopy (VPFM) amplitude and phase images after poling treatment with different voltages and relaxation durations of $\left(a_{1}\right),\left(b_{1}\right) 10$ min and $\left(a_{2}\right),\left(b_{2}\right) 30$ min for KNN-0.025LB and $\mathrm{KNN}-0.030 \mathrm{LB}$ ceramics 